\title{
The state of health of children of the first year of life, depending on the period of infection with cytomegalovirus
}

\begin{abstract}
The aim of the investigation: It was to study the state of health of children in the first year of life, depending on the period of infection with CMV.

Materials and methods: The study included 75 children and their mothers who were under observation from birth to one year of life. The evaluation of somatic, neurological status, neuropsychic and physical development, resistance of the organism was carried out. At the newborn, age of 3, 6 and 12 months, the serological status of children with antibody analysis was re-evaluated.

Results: Results was made the estimation of parameters of the health indicators, which includes in its analysis of indicators of neurodevelopment, physical development and resistance in children depending on presence or absence of CMVI at a birth and in the first 3months of life. Analysis of the correlations of the neuropsychic development and resistance showed reliable dependence of the early infection of cytomegalovirus infection and further development and the frequency and severity of morbidity of the child.
\end{abstract}

Conclusion: A significant effect of CMV on the neurodevelopment and resistance was found in the case of mild forms of congenital or early acquired infection in children.

Keywords: cytomegalovirus infection, neurodevelopment, physical development, the resistance of the organism, prenatal and early postnatal infection
Volume 3 Issue 3 - 2017

\author{
Khaletskaya OV,' Suslova MA, ${ }^{1,2}$ Fedyaeva \\ $\mathrm{AS},{ }^{2}$ latsyshina $\mathrm{EE}$ \\ 'Nizhny Novgorod State Medical Academy, Russia \\ ${ }^{2}$ Children’s City Clinical Hospital №I of Nizhni Novgorod, \\ Russia
}

Correspondence: Khaletskaya Olga, Head of the Department of Hospital Pediatrics, Nizhny Novgorod State Medical Academy, I0/I Minin and Pozharsky Square, Nizhny Novgorod, 603005, Russian Federation, Russia, Tel +79107945777, Email ovh14@mail.ru

Received: November 26, 2017 | Published: December 08, 2017

\section{Introduction}

Cytomegalovirus infection (CMVI) is an actual problem of perinatology, as it is one of the most frequent congenital infections and occurs in $0.2 \%$ to $2.5 \%$ of newborns.${ }^{1-4}$ Congenital CMVI can occur both in severe form, in which a lethal outcome is possible, and in the forms of low-symptom and asymptomatic variants. ${ }^{2,4-7}$ Low-symptom and asymptomatic forms often occur under the "masks" of various diseases. This is caused by the complexity of their diagnosis and the untimely appointment of therapy..$^{5,6,8-11}$ Thereby, it is interesting to study the effect of the persistence of cytomegalovirus (CMV) on the health status of children in the first year of life, which have the lowsymptomatic and asymptomatic forms of congenital or early postnatal infection. The aim of the research is to study the state of health of children in the first year of life, depending on the period of infection with CMV.

\section{Material and methods}

The study included 75 children and their mothers who were under observation from birth to one year of life. The study included newborns with a gestational age of more than 30 weeks, patients with low-symptom forms of CMVI or having a high risk of developing CMVI in accordance with anamnesis, but not having clinical signs of congenital CMVI during enrollment. The presence of suspicion of congenital malformations of the nervous system, hereditary pathology, metabolic diseases, signs of toxic-metabolic or traumatic damage to the nervous system, as well as signs of intrauterine infection of any other etiology served as exclusion criteria from the study.
The study was conducted in 3 phases. At the first stage on the neonatal period, when entering the in-patient department, in all children were evaluated: complaints and anamnesis; somatic and neurological status according to a standard procedure; laboratory and instrumental examination according to the standards of treatment of the underlying disease; as well as a laboratory examination at CMV with the use of Viral PCR with copy number and evaluation of the state of specific immunity with the determination of Ig M and Ig G with the definition of avidity to CMV.

At the second stage of the study, at the age of 3months, all the children were divided into two groups on the obtained basis of clinical and laboratory data.

Group I $(n=39)$ consisted of children who have laboratory confirmed CMV in the newborn period and/or in the first three months of life. The criteria for inclusion in group I(the presence of one of the signs):

a. The presence of IgM in the primary study; Appearance of Ig M in dynamics.

b. Appearance of Ig G in dynamics in previously seronegative children.

c. Decrease of avidity index (IA) Ig G and appearance of Ig G with low and transient IA to 3 months.

d. An increase in the Ig $\mathrm{G}$ titer by 4 or more times.

e. The detection of DNA pathogens in at least one biological fluid. 
In group II $(n=36)$ were children who did not have laboratory signs of CMV in the period of newborn and in the first three months of life. Criteria for inclusion in group II (the presence of not less than one of the signs):

a. Preservation of seronegativity.

b. Preservation in the dynamics of IgG with high IA without increasing the titer.

c. Preservation in the dynamics of Ig $\mathrm{G}$ with a transient IA in the absence of a tendency to decrease the IA and without a rise in the titer in the dynamics.

\section{d. DNA of pathogens was not detected.}

The third stage of the study included dynamic observation of children of both groups during the first year of life. For all patients were carried out the evaluation of somatic, neurological status, neuropsychic and physical development, resistance of the organism. At the age of 3, 6 and 12months, the serological status of children was analyzed again, with an analysis of antibody formation, evaluation of the somatic, neurological status, neurodevelopment and physical development assessment, and resistance of the organism.

Resistance was evaluated according to the frequency and severity of acute diseases that was transferred during the first year of life (Albitsky V Yu, Baranov AA, 1986). The severity of the disease was assessed by the severity of the clinical symptoms, the duration of the disease, the presence of complications, the need to prescribe antibiotic therapy, and also taking into account the time interval between episodes of acute diseases.

Children with high resistance had: the incidence of acute respiratory infections less than 4 times a year; duration of acute disease is less than 7days; use only symptomatic therapy for acute disease; the interval between episodes of acute diseases is not less than 2 months. In children with moderate resistance, the incidence of acute respiratory infections is 4-6times a year; duration of the disease up to 7-10days; antiviral drugs should be included in the therapy. Children with low resistance had: the incidence of acute respiratory infections more than 6times a year; with complications; duration of the disease is more than 10-14days; there is a need to prescribe antibiotic therapy, often in the hospital.

The method of the study was approved and approved by the Ethics Committee of the Nizhny Novgorod State Medical Academy of the Ministry of Health of the Russian Federation (28.12.2010). Statistical processing of data was carried out using parametric and nonparametric statistics using the application package of Microsoft Excel programs, licensed statistical programs "Statistica 10.0".

\section{Results}

Among the children included in the study, boys predominated $57.3 \%$ (43 patients), girls accounted for $42.7 \%$ (32 patients), full-term infants 64\%(48 children); 36\%(27 patients) were premature babies. The gestational age was $38.17 \pm 2.43$ weeks. In $12 \%(9$ children $)$ there was an intrauterine growth retardation. Birth body mass ranged from $1200 \mathrm{~g}$ to $4310 \mathrm{~g}$ and averaged $2988.39 \pm 585.98 \mathrm{~g}$, the average growth index was $49.76 \pm 3.14 \mathrm{~cm}$.

Clinical manifestations in the neonatal period in the children of the study group were differed in variety, and also had a nonspecific character. Among the clinical syndromes, the changes in the central nervous system ( $100 \%$ of patients) were the first in terms of frequency, on second place was the pathology of the cardiovascular system in the form of small cardiac abnormalities $(90.67 \%)$ and the atrial septal closure anomalies (the open oval window) $(76.0 \%)$ and in third place jaundice associated with disruption of bilirubin conjugation (77.33\%). There were no serious forms of infection. All clinical manifestations had a low-symptomatic character.

The neurodevelopment assessment showed that at the age of 3 months the children of the second group had significantly more normal development indices $\left(\chi^{2}=5.18, p=0.02\right)$. In 35patients $(89.70 \%)$ of the first group and $22(61.11 \%)$ of the second group there were changes in muscle tone and a tendency to disrupt the formation of the motor skills. At the age of 6 months, there was a significant deterioration in neurodevelopment in the first group of patients, so the normal indices of neurodevelopment have been in 2children $(5.13 \%)$ of the first group and $17(47.22 \%)$ of the second group $\left(\chi^{2}=10.6\right.$, $\mathrm{p}=0.001)$. Also among the children of the second group, there were significantly more frequent violations in the formation of motor skills $(\chi 2=4.00, \mathrm{p}=0.04)$.

This trend in the dynamics of the indices of neurodevelopment in children of both groups persisted till the age of one year. Normal parameters in development had been in 4 patients $(10.26 \%)$ of the first group and $17(47.22 \%)$ of the second group $\left(\chi^{2}=7.20, p=0.007\right)$. The delay in development was significantly more often detected in children of the second group than in the first group $\left(\chi^{2}=8.56, \mathrm{p}=0.003\right)$.

Analysis of physical development at age of the months did not show significant differences between the two groups of children $\left(\chi^{2}=0.01, p=0.90\right)$. At the age of 6 months, there were also no significant deviations in physical development $\left(\chi^{2}=0.37, p=0.54\right)$. At the age of the year, both in the first and in the second group, also patients with normal physical development indexes prevailed $-82.05 \%$ and $61.11 \%$, respectively $\left(\mathrm{x}^{2}=0.67, \mathrm{p}=0.42\right)$. Thereby, infection with CMV in the first three months of life has less impact on indicators of physical development.

Analysis of resistance in children at the age of one year showed that children of the first group were ill significantly more often and heavier in the first year of life. In the first group of children, only two $(5.13 \%)$ children had high resistance values, whereas in the second group - 13patients $(36.11 \%),\left(\chi^{2}=7.54, p=0.006\right)$. The average level of resistance was registered in $8(20.51 \%)$ patients first and in $12(33.33 \%)$ of the second group. Relatively often, low rates of resistance were observed among children of the first group $\left(\chi^{2}=4.50, \mathrm{p}=0.03\right)$. So $29(74.36 \%)$ of the children of the first group and $11(30.56 \%)$ of the second group had low rates of resistance.

\section{Discussion}

The study of health indicators in children on the first year of life with a proven not severe congenital and/or early postnatal form CMVI showed a significant deterioration in the neurodevelopmental and resistance rates in these children compared to patients who did not have serological signs of the CMV infection at birth and in the first three months of life. The indicators of physical development were less dependent of CMVI, which suggests a greater influence of other factors on this indicator (the nature of feeding, constitutional features). The revealed reliable influence of CMV on the neurodevelopment and resistance in infants with not severe forms of infection leads to the need for a survey to clarify the nature of the infection process caused 
by the cytomegalovirus in all children of high risk perinatal infection, regardless of the presence or absence clinical symptoms.

Thus, perinatal and early postnatal CMV infection in the first three months of life is one of the significant causes of neurodevelopmental delayeds and immune disorders in children in their first year of life. The obtained results are confirmed by literature data. ${ }^{1-3}$ It is known that CMV is able to cause morphological changes in the neural tissue due to neurotropism and the ability to persistence, which becomes especially important at an early age, when the most intensive growth processes occur. These morphological changes are manifested as a violation of the processes of myelination of nerve fibers, especially the cortex, necrosis of individual neurons, proliferation of glia. Also, a number of authors have demonstrated the ability of CMV to influence the state of cerebral vessels, which is probably associated with the epitheliotropy of the cytomegalovirus. The negative influence of CMV on the cerebral vessels, in turn, can strengthen the effect of other damaging factors acting on nerve cells, for example hypoxia, which can also have an impact on the development of the child in the future.

\section{Conclusion}

i. Perinatal and early postnatal CMVI in the first three months of life is associated with a delay of neurodevelopmental in children in the first year of life.

ii. The indicators of physical development are less dependent on infection of CMV. The growth-weight indicators are more influenced by constitutional factors, the nature of feeding, etc.

iii. Perinatal and early postnatal CMVI in the first three months of life leads to a violation of resistance in the first year of life. Children who have serological signs of active cytomegalovirus infection in the first three months are significantly more often and more seriously ill with acute illnesses in the first year of life.

iv. Children with laboratory confirmed perinatal and early postnatal CMVI in the first three months of life, even in low-symptom and asymptomatic forms, need dynamic monitoring of clinical indices and indicators of the state of specific humoral immunity to $\mathrm{CMV}$.

\section{Acknowledgements}

My research project has no financial assistance. Payment at the expense of authors own funds.

\section{Conflicts of interest}

The authors declare that there is no conflict of interest.

\section{References}

1. Orekhov KVM. Intrauterine infections and pathology of newborns. Russia: Medpraktika; 2002. 252 p.

2. Protocols for diagnosis, treatment and prevention of intrauterine infections in newborns. 2nd ed. Moscow: GOU VUNMTS MH RF; p. 104.

3. Isakov VA. Herpesvirus infection: recommendations for doctors. 2006. $96 \mathrm{p}$.

4. Kenneson A, Cannon MJ. Review and meta-analysis of the epidemiology of congenital cytomegalovirus (CMV) infection. Rev Med Virol. 2007;17(4):253-276.

5. Chikina TA. Early and late neurologic complications in children with a latent form of cytomegalovirus infection. Questions of practical pediatrics. 2007;2(3):18-20.

6. Pass RF, Fowler KB, Boppana SB, et al. Congenital cytomegalovirus infection following first trimester maternal infection symptoms at birth and outcome. J Clin Virol. 2006;35(2):216-220.

7. Dollard SC, Grosse SD, Ross DS. New estimates of the prevalence of neurological and sensory sequelae and mortality associated with congenital cytomegalovirus infection. Rev in Medical Virology. 2007;17(5):355-363.

8. Noyola DE, Demmler GJ, Nelson CT, et al. Early predictors of neurodevelopmental outcome in symptomatic congenital cytomegalovirus infection. J Pediatr. 2001;138(3):325-331.

9. Fedyaeva A. Indices of psychomotor development of children in the first year of life depending on the state of specific immunity to herpetic infections. Europaediatrics. Russia; 2009.

10. Fedyaeva AS, Khaletskaya OV, Shutkova Fedyaeva A Yu, et al. The state of specific immunity to herpes infections and indices of physical and neuropsychic development in young children. Medicinskiy al'manah. 2009;3(8):156-159.

11. Fedyaeva AS, Khaletskaya OV, Shutkova Fedyaeva A Yu. Comprehensive assessment of the state of health of young children infected with herpes viruses in the first half of the year. Medicinskiy al'manah. 2010;2(11):84-88. 